\title{
1 Perforin-2 limits pathogen proliferation at the maternal-fetal interface
}

3 Petoria Gayle*, Vanessa McGaughey*, Rosmely Hernandez*, Marina Wylie*t, Rachel C.

4 Colletti*†, Ka Lam Nguyen*t, Marshall Arons*, Laura Padula*, Natasa Strbo*, Kurt Schesser*

5

6 *Department of Microbiology \& Immunology, University of Miami Miller School of Medicine,

7 Miami, FL, USA

8

9 Corresponding Author: Kurt Schesser, Department of Microbiology and Immunology, University

10 of Miami Miller School of Medicine, 1600 NW 10 ${ }^{\text {th }}$ Avenue, Miami, FL 33136.

11 kschesser@med.miami.edu

12

13 † Current addresses: Uniformed Services University of the Health Sciences, Bethesda, MD (MW);

14 Philadelphia College of Osteopathic Medicine, Philadelphia, PA (RCC); The University of Illinois

15 at Urbana-Champaign, Champaign, IL (KLN).

16

17 PG and KS conceived the project, designed the experiments, and interpreted the data. All 18 experiments and data collection were performed by PG with assistance by VM, RH, MW, RCC,

19 KLN, MA, and KS. Contributions to the development of methodologies were made by LP and NS.

20 The manuscript was primarily written by PG with assistance by KS. 


\section{Abstract:}

22 Placental immune responses are highly regulated to strike a balance between protection and

23 tolerance. For relatively mild infections, protection encompasses both the mother and fetus;

24 however, during worsening conditions, protection becomes exclusively reserved for the mother.

25 Previously, we and others have shown that the host factor Perforin-2 plays a central role in

26 protecting mice and cells against infection. Here, we analyzed Perforin-2 activity in the mouse

27 placenta to determine whether Perforin-2 plays a similarly protective role. We show that Perforin-

282 is critical for inhibiting Listeria monocytogenes colonization of the placenta and fetus and that

29 this protection is due to both maternal and fetal-encoded Perforin-2. Perforin-2 mRNA is readily

30 detectable in individual immune cells of the decidua and these levels are further enhanced

31 specifically in decidual macrophages during high-dose infections that result in fetal expulsion.

32 Unexpectedly, inductive Perforin-2 expression in decidual macrophages did not occur during

33 milder infections in which fetal viability remained intact. This pattern of expression significantly

34 differed from that observed in splenic macrophages in which inductive Perforin-2 expression was

35 observed in both high and mild infection conditions. In the placenta, inductive Perforin-2

36 expression in decidual macrophages was co-incident with their polarization from a M2 to M1

37 phenotype that normally occurs in the placenta during high-burden infections. Our results suggest

38 that Perforin-2 is part of a host response that is protective either for both the mother and fetus in

39 milder infections or exclusively for the mother during high-dose infections. 
Introduction:

41 The balance between host defense and tolerance during pregnancy is achieved both by the

42 modulation of the maternal immune system towards the semi-allogenic fetus as well as through

43 the barrier function of the placenta. Despite these safeguards, infections contribute approximately

$4425 \%$ of stillbirths in the United States, largely due to the ability of specific pathogens, including

45 Listeria monocytogenes, to colonize the placenta (McClure et al. 2010). L. monocytogenes is

46 widely used in hematogenous infection models to study host-pathogen interactions in the placenta

47 (Lamond and Freitag 2018). While these models have advanced our understanding of the

48 pathogenesis of placental infections, there still remains the need to further understand the

49 mechanisms of immune defense in coordination with fetal tolerance during placental infection.

50 Numerous studies have shown that infections during pregnancy can disrupt the highly

51 controlled inflammatory response and result in pregnancy complications such as miscarriages or

52 spontaneous abortion (Kim et al. 2005; Mor et al. 2017; Rodrigues-Duarte et al. 2018). These

53 pregnancy related complications are commonly attributed to the activation of the innate immune

54 response. Specifically, macrophages of the decidua, the maternal component of the placenta, have

55 been shown to be highly dynamic during pregnancy. These cells change and respond to the

56 inflammatory environment of the placenta expressing characteristics of the classically activated

57 phenotype, M1, in early and late pregnancy and resembling the M2 phenotype during the mid-

58 stage of pregnancy (Shapouri-Moghaddam et al. 2018; Zhang et al. 2017). Excessive levels of pro-

59 inflammatory M1 macrophages have been linked to abnormal pregnancy outcomes including pre-

60 term labor and fetal mortality (Jena et al. 2019; Svensson-Arvelund and Ernerudh 2015; Wang et

61 al. 2011; Xu et al. 2016). It has also been shown that L. monocytogenes can trigger M1 polarization

62 in the placenta (Benoit et al. 2008). Studying the role of innate immune factors within the placenta 
63 is important to enhance our understanding of how to prevent the devastating effects of infection

64 during pregnancy.

65 In vertebrates there are three pore-forming factors that protect against microbial pathogens.

66 Complement component 9 (C9) and Perforin-1 possess membrane-attack-complex-perforin

67 (MACPF) domains that mediate their polymerization and pore formation. The third and most

68 recently identified vertebrate MACPF-containing factor, Perforin-2, is found in the earliest

69 evolved animals, and is ancestral to C9 and Perforin-1 (D'Angelo et al. 2012). Unlike the

70 Complement Factors and Perforin-1 that are secreted from cells, Perforin-2 is an integral

71 membrane protein. In human and mouse cells Perforin-2 mRNA is constitutively present in

72 macrophages and is induced in fibroblastic cells following infection or exposure to inflammatory

73 signals (McCormack et al. 2013). Perforin-2 plays a major role in protecting mice from Listeria,

74 Salmonella, Staphylococcus, and Yersinia infections (McCormack et al. 2015a; 2015b; 2016). In

75 vitro, Perforin-2 restricts intracellular L. monocytogenes proliferation by a $\mathrm{pH}$-dependent

76 mechanism in both primary peritoneal macrophages and fibroblastic cells suggesting that the rapid

77 development of listeriosis in Perforin-2 -/- mice is due to defects in cellular killing activity

78 (McCormack et al. 2016). Recently it has been shown that Perforin-2 directly impacts type I

79 interferon signaling by physically interacting with the IFN- $\alpha$ and $-\beta$ receptors 1 and 2 (McCormack

80 et al. 2020). Whether the bactericidal activity of Perforin-2 involves the interferon signaling

81 machinery remains to be determined.

82 Here we tested whether Perforin-2 plays a protective role in limiting colonization of $L$.

83 monocytogenes in the mouse placenta. Additionally, we analyzed Perforin-2 mRNA expression in

84 individual cells of the placenta following infection to determine whether Perforin-2 expression

85 levels change during infection. 
Materials and Methods:

87 Mice, microbes, and infections

88 Wild-type BALB/c and C57BL/6 Perforin-2 +/+ and Perforin-2 -/- littermates (McCormack et al.

89 2015a) were bred in the animal care facility at the University of Miami Miller School of Medicine,

90 Miami, FL. All animal procedures were approved by the Institutional Animal Care and Use

91 Committee, University of Miami Miller School of Medicine (protocol 19-075). Animals were

92 housed under a circadian cycle (12hr light/12hr dark cycle). Virgin female mice were mated

93 between 6 - 12 weeks of age, and checked daily for estrous stage and copulatory plugs. Presence

94 of a plug was denoted as gestation day (GD) 0.5. Weight gain was monitored on GD 4.5 through

95 GD 12.5 to confirm pregnancy. L. monocytogenes (10403S) were grown in brain heart infusion

96 media at $37{ }^{\circ} \mathrm{C}$ with vigorous shaking to mid-log phase. Mice at GD 12.5 were infected

97 intravenously with doses as indicated in figure legends. At 44 hours post infection (hpi), mice were

98 humanely euthanized and uterine horns, livers, and spleens were removed and processed for either

99 single cell analysis (see below) or colony forming unit (CFU) assays. For CFU assays, placentas

100 (including decidual tissue), fetuses, and livers were further dissected and homogenized using a fine

101 wire mesh to grind the tissues in sterile water containing $0.05 \%$ Tween. The resulting tissue

102 homogenates were diluted and plated on Luria Broth agar to determine L. monocytogenes titers. In

103 the experiments using heterogenic matings, fetal tissue homogenates were used for genotyping.

104 Preparation of single-cell suspensions

105 Following their removal, uterine horns were dissected to remove individual fetal-placental units

106 (FPU), each FPU was further dissected to isolate decidual tissue. Livers (minced) and pooled

107 deciduae were incubated in $2 \mathrm{mg} / \mathrm{mL}$ collagenase $\mathrm{D}$ (Roche) at $37^{\circ} \mathrm{C}$ for $30-40$ minutes with

108 agitation. The resulting cell suspensions were then washed with cold IMDM (Life Technologies) 
$109+10 \%$ heat-inactivated FBS (Sigma-Aldrich), passed through a $70 \mu \mathrm{m}$ filter followed by passaging

110 through a $40 \mu \mathrm{m}$ filter (VWR International, Radnor, PA). Spleens were gently homogenized

111 through $70 \mu \mathrm{m}$ filters and similarly washed and passed through $40 \mu \mathrm{m}$ filters. The resulting single

112 cells were then centrifuged at $500 \mathrm{~g}$ for 5 min at $4^{\circ} \mathrm{C}$, treated with ACK Lysing Buffer (Life

113 Technologies Corporation) for 5 minutes to remove red blood cells, and finally washed with

114 IMDM $+10 \%$ FBS. Total viable cells were determined using the Vi-Cell XR Cell Viability

115 Analyzer (Beckman Coulter).

116 Single cell analysis

117 Isolated cells were washed in FACS staining buffer and incubated with anti-CD16/32 (clone

118 2.4G2) to block FcRs for 10 mins followed by an incubation with Live/Dead fixable yellow dead

119 cell stain (ThermoFisher) and fluorescent-conjugated monoclonal antibodies (mAbs) for 30 min.

120 The following anti-mouse mAbs were used for analysis: CD45 (30-F11; BioLegend), CD3 (17A2;

121 BioLegend), F4/80 (BM8; BioLegend), CD11b (M1/70; BioLegend), Ly6C (HK1.4; BioLegend),

122 Ly6G (1A8; BioLegend), CD335 (29A1.4; BioLegend), MHC II (M5/114.15.2; BD Biosciences),

123 CD206 (C068C2; BioLegend). Depending on the experiment, samples were either immediately

124 analyzed by flow cytometry using a Sony SP6800 Spectral Analyzer or further processed to

125 determine Perforin-2 mRNA levels. Branched oligonucleotide signal amplification was used to

126 determine Perforin-2 mRNA levels in individual cells (PrimeFlow; ThermoFisher Scientific).

127 Briefly, single cell suspensions were stained for surface antigens as described above, then fixed,

128 permeabilized, and incubated with probes specific for Perforin-2 transcripts (Assay Id: VB1-

129 20172-PF; ThermoFisher Scientific). Cells were then subjected to a series of signal amplification

130 cycles and then analyzed by flow cytometry as described above using FlowJo software (BD

131 Biosciences). 


\section{Results:}

133 Perforin-2 limits pathogen colonization at the maternal-fetal interface

134 Pregnant wild-type and isogenic Perforin-2-deficient (Perforin-2 -/-) mice were infected

135 intravenously on gestation day (GD) 12.5 (i.e., mid-gestation) with L. monocytogenes. At 44 hours

136 post infection, dams were humanely euthanized and livers and fetal placental units (FPU) were

137 evaluated for L. monocytogenes by colony forming unit (CFU) assay. In infected Perforin-2 +/+

138 dams, $54 \%(12 / 22)$ of the placentas and 14\% (3/22) of fetuses possessed detectable levels of $L$.

139 monocytogenes (Fig. 1). Dosages that result in approximately 50\% of placentas in Perforin-2 +/+

140 mice becoming infected will henceforth be referred to as placental dose 50 (PD50). In contrast,

141 Perforin-2 -/- mice harboring comparable levels of L. monocytogenes in the liver as Perforin-2

$142+/+$ dams, 88\% (15/17) of the placentas and 71\% (12/17) of fetuses possessed detectable levels of

143 L. monocytogenes. These results suggest that Perforin-2 may play a significant role in limiting $L$.

144 monocytogenes colonization of the placenta and fetus.

146 Maternal and fetal-derived Perforin-2 contributes to protection against infection in the placenta

147 The placenta is a chimeric organ that consists of maternally-derived tissue and fetal-derived

148 trophoblast cells. A heterogenic mating strategy was used to evaluate the specific contributions of

149 maternal- and fetal-derived Perforin-2 in limiting L. monocytogenes colonization in the placenta.

150 Initially, Perforin-2 -/- female mice were crossed to Perforin-2 +/- males (generating

151 approximately 50\% Perforin-2 +/- and 50\% Perforin-2 -/- fetuses) and at GD 12.5, dams were

152 infected with $\mathrm{PD}_{50}$ L. monocytogenes and analyzed as described above. A representative dam is

153 shown in which the infection burdens of FPUs containing Perforin-2 +/- fetuses are generally

154 lower than FPUs containing Perforin-2 -/- fetuses (Fig. 2A). In compiled data from 5 dams, the 
155 placentas associated with Perforin-2 +/- fetuses (designated as $\mathrm{T}(+/-)$ ) had significantly lower

156 infection burdens compared to placentas associated with Perforin-2 -/- fetuses (designated as T(-

157 /-)). A similar reduction in infection burden was observed in Perforin-2 +/- fetuses compared to

158 Perforin-2 -/- fetuses (Fig. 2B) indicating that fetal-derived Perforin-2 protects the placenta from

159 infection. In reciprocal matings, in which Perforin-2 +/- female mice were crossed with Perforin-

1602 -/- males and infected at GD 12.5, a more modest protective effect of fetal-derived Perforin-2

161 was observed (Fig. 2C), possibly indicating that maternal-derived Perforin-2 partially masks the

162 protective effect of fetal-derived Perforin-2. Collectively these data show that both maternal- and

163 fetal-derived Perforin-2 contribute to protecting the placenta from being colonized by a bloodborne

164 pathogen.

Perforin-2 expression is induced in placental immune cells following infection

167 The maternal component of the placenta, the decidua, is the initial colonization site of various

168 bloodborne pathogens including L. monocytogenes (Rizzuto et al. 2017). We initially analyzed the

169 cellular composition of the decidua and the liver in uninfected and infected mid-gestation mice.

170 Previously we and others showed that the immune cell composition in the liver in non-pregnant

171 mice undergoes substantial changes following L. monocytogenes infection (Gregory et al. 2002;

172 Blériot et al. 2015; Gayle et al. 2019). There was a similar pattern of changes in the liver observed

173 in mid-gestation pregnant mice following infection, including a marked disappearance of resident

174 macrophages $\left(\mathrm{CD} 11 \mathrm{~b}^{\mathrm{lo}} / \mathrm{F} 4 / 80^{+}\right)$and the infiltration of inflammatory monocytes and neutrophils

175 (CD11b $\left.{ }^{+} / \mathrm{F} 4 / 80^{-}\right)$(Fig. 3A). In contrast, in the decidua of the same mice, there were no significant

176 changes in the resident macrophages $\left(\mathrm{CD} 11 \mathrm{~b}^{+} / \mathrm{F} 4 / 80^{+}\right)$or other cell types following infection (Fig.

177 3B). From GD 12.5 - 14.5 pregnant mice approximately a million cells are typically isolated from 
178 individual decidua of which $\sim 20 \%$ are $\mathrm{CD}_{4} 5^{+}$. The $4 \times 10^{5} \mathrm{CD} 45^{+}$cells per decidua is composed

179 of approximately 20\% CD11b $/ \mathrm{F}^{-} \mathrm{C}^{-}$(primarily NK cells), 30\% CD11b ${ }^{+} / \mathrm{F}^{2} 40^{-}$(monocytes and 180 neutrophils), and 40\% CD11b ${ }^{+} / \mathrm{F} 480^{+}$(macrophages).

181 Perforin-2 mRNA levels were assayed in individual decidual cells to determine both cell 182 type-specific expression and whether expression levels change following infection. To ensure that 183 all placentas within each pregnant mouse became colonized by L. monocytogenes during the $44 \mathrm{hr}$ 184 infection period, the doses used for these experiments will be referred to as placental dose $100 \%$ $185\left(\mathrm{PD}_{100}\right)$ that are 2- to 4-fold higher than the 'low-dose' experiments shown in Figs. 1 and 2. As 186 described earlier, pregnant mice (GD 12.5) were either left uninfected or infected with $L$. 187 monocytogenes and following 44 hours of infection, FPUs were collected and placentas were 188 further dissected to isolate decidual cells. Pooled decidual cells from each individual dam were 189 analyzed for cell surface markers and Perforin-2 mRNA levels. In uninfected dams, Perforin-2 190 mRNA was readily detected in $\mathrm{CD}^{4} 5^{+}$decidual cells and this signal was enhanced 2- to 3-fold in $191 \mathrm{CD}^{4} 5^{+}$decidual cells isolated from infected dams (Fig. 4A, B). A similar 2- to 3-fold infection192 dependent increase in Perforin-2 mRNA levels was also observed in $\mathrm{CD}^{4} 5^{+}$splenic cells derived 193 from the same mice (Fig. 4C).

Of the three major CD45 ${ }^{+}$subsets found in the GD 12.5 decidua of BALB/c mice (see Fig.

195 3B), infection-induced enhancement of Perforin-2 mRNA levels occurred primarily in the

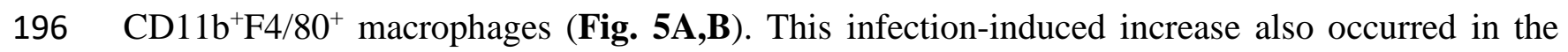
$197 \mathrm{CD} 45^{+}$splenic macrophages derived from the same mouse (Fig. 5C). In addition, Perforin-2 198 mRNA was also specifically induced in infected decidual and splenic CD11b ${ }^{+} \mathrm{F} 4 / 80^{+}$macrophages 199 isolated from C57BL/6 mice (Fig. 5D-F). These data show that Perforin-2 mRNA levels increase 200 in immune cells of the decidua following infection and that this increase primarily occurs in 
macrophages.

202

203 Reduced infection dosages result in modest changes in Perforin-2 mRNA levels in decidual 204 macrophages

205 As noted earlier, the Perforin-2 expression experiments shown in Figs. 4 and 5 used infective doses

$206\left(\mathrm{PD}_{100}\right)$ that resulted in all placentas becoming colonized with L. monocytogenes by 44 hpi. 207 Unexpectedly, when these experiments were performed with 2- to 4-fold lower doses of $L$. 208 monocytogenes in which approximately 50\% of placentas had become colonized by $L$. 209 monocytogenes by 44 hpi (PD50), there was little to no difference in Perforin-2 mRNA levels

210 between decidual macrophages isolated from uninfected and infected GD 12.5 pregnant mice (Fig.

211 6A,B). In contrast, in the same mice Perforin-2 mRNA levels were significantly elevated in splenic

212 macrophages isolated from infected mice compared to uninfected mice (Fig. 6C). A similar pattern

213 of Perforin-2 expression in decidual and splenic macrophages was observed in pregnant GD 12.5

214 C57BL/6 mice infected at PD50 (Fig. 6D,E). These findings suggest that in the placenta there is a

215 bacterial dose-dependent induction of Perforin-2 mRNA.

217 Perforin-2 mRNA levels are elevated in M1 decidual macrophages

218 In humans and mice, decidual macrophages at mid-gestation are primarily of the M2 phenotype 219 and polarization of these macrophages to the inflammatory M1 phenotype is associated with a 220 variety of complications that can lead to premature pregnancy termination (Brown et al. 2014; Jena

221 et al. 2019; Svensson-Arvelund and Ernerudh 2015; Xu et al. 2016). To determine the infection-

222 specific phenotype of decidual macrophages in our model, pregnant GD 12.5 mice were either left

223 uninfected or infected with L. monocytogenes at $\mathrm{PD}_{100}$. Following their isolation, decidual cells 
224 were stained for M1 and M2 specific markers and analyzed by flow cytometry. Macrophages were

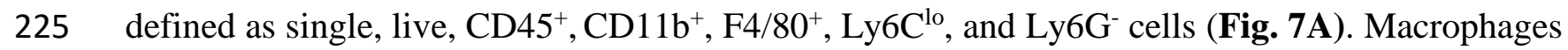

226 were then further classified as either M1 $\left(\mathrm{CD}^{-} \mathrm{C}^{-} \mathrm{MHCII}^{\mathrm{hi}}\right)$ or M2 (CD206 $\left.{ }^{+} \mathrm{MHCII}^{\mathrm{lo}}\right)$ in

227 uninfected and infected dams. Consistent with previously published findings cited above, decidual

228 macrophages isolated from uninfected dams were primarily M2. In contrast, decidual macrophages

229 isolated from infected dams contained lower frequencies of CD206 ${ }^{+}$cells and higher frequency of

230 MHCII cells indicative of a M1-skewed phenotype (Fig. 7). When GD 12.5 mice were infected

231 with a reduced dose $\left(\mathrm{PD}_{50}\right)$ of L. monocytogenes, the M1/M2 distribution of decidual macrophages

232 at 44 hpi did not appreciatively differ from that observed in decidual macrophages isolated from

233 uninfected GD 12.5 mice (not shown).

234 Decidual macrophages isolated from uninfected dams at mid-term pregnancy (GD 12.5)

235 were stained for M1 and M2 specific markers as described above and subsequently analyzed for

236 Perforin-2 mRNA levels. In every pregnant mouse examined, Perforin-2 mRNA levels were

237 notably elevated in M1 decidual macrophages compared to M2 decidual macrophages (Fig. 8).

238 Collectively, these data show that at high pathogen burdens M1 macrophages with heightened

239 Perforin-2 mRNA levels predominate in the placenta. 
Discussion:

241

A unique challenge in both the operation as well as the study of immune responses

242 occurring during pregnancy is that protection for the host may not always encompass the fetus.

243 While the placenta acts as an immunological and physical barrier for fetal protection, it is

244 susceptible to pathogens capable of colonizing this tissue and, under worsening conditions, can

245 reorient towards an exclusively maternal protective response (Robbins and Bakardjiev 2012;

246 Zeldovich and Bakardjiev 2012; Bonney and Johnson 2019; Jena et al. 2019). Decidual

247 macrophages have been shown to be critical for the plasticity of the placental response in being

248 important for both the maintenance of fetal tolerance as well as responding to inflammatory and/or

249 infectious conditions that can result in immune activation against the fetus (Brown et al. 2014;

250 Wang et al. 2018).

251 We show here that Perforin-2 plays a significant role during L. monocytogenes infection in

252 the placenta and fetus. We additionally found that fetal-derived Perforin-2 in the placenta

253 contributes to limiting L. monocytogenes infection, although there is a dominance in regards to the

254 maternal expression of Perforin-2. These findings are consistent with published data showing that

255 the maternal-derived decidua is the initial site of infection and the first line of defense in

256 bloodborne L. monocytogenes infections (Rizzuto et al. 2017). In analysis of Perforin-2 mRNA

257 levels in individual decidual cells, we found that $\mathrm{CD}_{4} 5^{+}$cells possessed abundant transcripts, and

258 specific to decidual macrophages, these levels became elevated in infections with high pathogen

259 loads. This inductive Perforin-2 expression in decidual macrophages correlated with their

260 polarization from the tolerogenic M2 phenotype to the inflammatory M1 phenotype. In these

261 experiments with high pathogen loads (i.e., $\mathrm{PD}_{100} ; 5-10 \times 10^{5} \mathrm{CFU}$ ), dams often displayed typical

262 signs of listeriosis at 44 hpi including ruffled fur, hunched backs, and slowed movement. Also 
263 there would occasionally be FPU observed in the cage housing presenting with darkened uterine

264 horns and loss of fetal structures in remaining fetuses, all indicative of maternal immune activation

265 (MIA) (Goldstein et al. 2017). In contrast, there was little to no inductive Perforin-2 expression in

266 decidual macrophages in infections with relatively lower pathogen loads (i.e., $\mathrm{PD}_{50} ; 2$ x $10^{5}$ CFU)

267 in which neither disease symptoms nor fetal expulsions occur. These findings indicate that

268 inductive Perforin-2 expression in deciduae is associated with a pathological shift in macrophage 269 phenotype.

270 In understanding Perforin-2 expression in decidual macrophages, it is worth noting that

271 there are three immunological stages of pregnancy (Mor et al. 2017). At the early stage of

272 pregnancy, when the blastocyst is implanted into the uterine wall, there is increased inflammation

273 and a $T h_{1}$-biased environment, during which there is a predominance of M1 macrophages in the

274 decidua. It has been shown that this inflammation is not in response to the invading fetal antigens

275 but that there is an active recruitment by the trophoblast cells in an effort to educate immune cells

276 towards fetal tolerance. The mid-stage of pregnancy is a period of fetal growth and when fetal

277 tolerance is the most important, as pathological conditions that lead to MIA can result in a breach

278 of fetal tolerance and therefore fetal rejection (Mor et al. 2017). In line with this, the mid-term of

279 pregnancy represents an anti-inflammatory state, generating a tolerogenic $\mathrm{Th}_{2}$-type immune

280 environment in which M2 macrophages dominate within deciduae. Lastly, the late stage of

281 pregnancy again requires inflammation that is necessary for labor induction and is characterized

282 by a $\mathrm{Th}_{1}$ immune response in which again there is a predominance of M1 macrophages.

283 Pathogens capable of crossing the placental barrier can take advantage of the anti-

284 inflammatory microenvironment of the placenta during the mid-stage of pregnancy. However,

285 once a certain threshold is reached and MIA occurs, fetal tolerance can be disrupted and result in 
286 pregnancy termination (Bonney and Johnson 2019). Perforin-2 serves both a protective function

287 in sub-MIA infections (i.e., as shown in the PD50 experiments [Figs. 1 and 2]) as well as becoming

288 elevated during infections in which MIA is triggered (Fig. 5). Collectively, our investigation may

289 indicate that Perforin-2 is a component that acts to protect either both the mother and fetus (sub-

290 MIA infections) or exclusively the mother (MIA infections). 
References:

292 Benoit M, Desnues B, Mege JL. Macrophage polarization in bacterial infections. J Immunol.

2932008 Sep 15;181(6):3733-9.

294

295 Blériot C, Dupuis T, Jouvion G, Eberl G, Disson O, Lecuit M. Liver-

296 resident macrophage necroptosis orchestrates type 1 microbicidal inflammation and type-2-

297 mediated tissue repair during bacterial infection. Immunity. 2015 Jan 20;42(1):145-58

298

299 Bonney EA, Johnson MR. The role of maternal T cell and macrophage activation in preterm

300 birth: Cause or consequence? Placenta. 2019 Apr;79:53-61.

301

302 Brown MB, von Chamier M, Allam AB, Reyes L. M1/M2 macrophage polarity in normal and 303 complicated pregnancy. Front Immunol. 2014 Nov 24;5:606.

Gayle P, Freitag NE, Strbo N, Schesser K. Using a Bacterial Pathogen to Probe for Cellular and 306 Organismic-level Host Responses. J Vis Exp. 2019 Feb 22;(144).

308 Goldstein JA, Norris SA, Aronoff DM. DOHaD at the intersection of maternal immune 309 activation and maternal metabolic stress: a scoping review. J Dev Orig Health Dis. 2017 310 Jun;8(3):273-283.

312 Gregory SH, Cousens LP, van Rooijen N, Döpp EA, Carlos TM, Wing EJ.Complementary

313 adhesion molecules promote neutrophil-Kupffer cell interaction and the elimination of bacteria

314 taken up by the liver. J Immunol. 2002 Jan 1;168(1):308-15.

316 Jena MK, Nayak N, Chen K, Nayak NR.

317 Role of Macrophages in Pregnancy and Related Complications.

318 Arch Immunol Ther Exp (Warsz). 2019 Oct;67(5):295-309. 
320 Kim S, Lee DS, Watanabe K, Furuoka H, Suzuki H, Watarai M.Interferon-

321 gamma promotes abortion due to Brucella infection in pregnant mice. BMC Microbiol. 2005

322 May 4;5:22.

323

324 Lamond NM, Freitag NE. Vertical Transmission of Listeria monocytogenes: Probing the Balance

325 between Protection from Pathogens and Fetal Tolerance. Pathogens. 2018 May 25;7(2).

326

327 Rizzuto G, Tagliani E, Manandhar P, Erlebacher A, Bakardjiev AI. Limited Colonization

328 Undermined by Inadequate Early Immune Responses Defines the Dynamics of Decidual

329 Listeriosis. Infect Immun. 2017 Jul 19;85(8).

Li W, Chang Y, Liang S, Zhong Z, Li X, Wen J, Zhang Y, Zhang J, Wang L, Lin H, Cao X,

332 Huang H, Zhong F. NLRP3 inflammasome activation contributes to Listeria monocytogenes-

333 induced animal pregnancy failure. BMC Vet Res. 2016 Feb 24;12:36.

McClure EM, Dudley DJ, Reddy UM, Goldenberg RL. Infectious causes of stillbirth:

336 a clinical perspective. Clin Obstet Gynecol. 2010 Sep;53(3):635-45.

McCormack, R. and Eckhard R. Podack. 2015. "Perforin-2/Mpeg1 and Other Pore-Forming

341 McCormack R, Bahnan W, Shrestha N, Boucher J, Barreto M, Barrera CM, Dauer EA, Freitag

342 NE, Khan WN, Podack ER, Schesser K. Perforin-2 Protects Host Cells and Mice by Restricting

343 the Vacuole to Cytosol Transitioning of a Bacterial Pathogen. Infect Immun. 2016 Mar

344 24;84(4):1083-1091.

346 McCormack R, de Armas LR, Shiratsuchi M, Ramos JE, Podack ER. Inhibition of intracellular 347 bacterial replication in fibroblasts is dependent on the perforin-like protein (perforin-2) encoded 348 by macrophage-expressed gene 1. J Innate Immun. 2013;5(2):185-94. 
350 McCormack RM, de Armas LR, Shiratsuchi M, Fiorentino DG, Olsson ML, Lichtenheld MG,

351 Morales A, Lyapichev K, Gonzalez LE, Strbo N, Sukumar N, Stojadinovic O, Plano GV,

352 Munson GP, Tomic-Canic M, Kirsner RS, Russell DG, Podack ER. Perforin-2 is essential for

353 intracellular defense of parenchymal cells and phagocytes against pathogenic bacteria. Elife.

3542015 Sep 24;4. pii: e06508.

McCormack R, Hunte R, Podack ER, Plano GV, Shembade N. An Essential Role for Perforin-2 in Type I IFN Signaling. J Immunol. 2020 Apr 15;204(8):2242-2256

Money KM, Barke TL, Serezani A, Gannon M, Garbett KA, Aronoff DM, Mirnics K.

Gestational diabetes exacerbates maternal immune activation effects in the developing brain.

Mol Psychiatry. 2018 Sep;23(9):1920-1928.

Mor G, Aldo P, Alvero AB. The unique immunological and microbial aspects of pregnancy. Nat Rev Immunol. 2017 Aug;17(8):469-482.

Rizzuto G, Tagliani E, Manandhar P, Erlebacher A, Bakardjiev AI.

367 Limited Colonization Undermined by Inadequate Early Immune Responses Defines the Dynamic s of Decidual Listeriosis. Infect Immun. 2017 Jul 19;85(8).

Robbins JR, Bakardjiev AI. Pathogens and the placental fortress. Curr Opin Microbiol. 2012

373 Rodrigues-Duarte L, Pandya Y, Neres R, Penha-Gonçalves C. Fetal and Maternal Innate

374 Immunity Receptors Have Opposing Effects on the Severity of Experimental Malaria in

375 Pregnancy: Beneficial Roles for Fetus-Derived Toll-Like Receptor 4 and Type I Interferon

376 Receptor 1. Infect Immun. 2018 Apr 23;86(5).

378 Shapouri-Moghaddam A, Mohammadian S, Vazini H, Taghadosi M, Esmaeili SA, Mardani F,

379 Seifi B, Mohammadi A, Afshari JT, Sahebkar A. Macrophage plasticity, polarization, and function in health and disease. J Cell Physiol. 2018 Sep;233(9):6425-6440 
382 Strbo N, Pastar I, Romero L, Chen V, Vujanac M, Sawaya AP, Jozic I, Ferreira ADF, Wong LL,

383 Head C, Stojadinovic O, Garcia D, O'Neill K, Drakulich S, Taller S, Kirsner RS, Tomic-Canic

384 M. Single cell analyses reveal specific distribution of anti-bacterial molecule Perforin-2 in

385 human skin and its modulation by wounding and Staphylococcus aureus infection.

386 Exp Dermatol. 2019 Mar;28(3):225-232.

388 Svensson-Arvelund J, Ernerudh J. The Role of Macrophages in Promoting and Maintaining

389 Homeostasis at the Fetal-Maternal Interface. Am J Reprod Immunol. 2015 Aug;74(2):100-9

391 Wang WJ, Hao CF, Lin QD.

392 Dysregulation of macrophage activation by decidual regulatory T cells in unexplained recurrent 393 miscarriage patients. J Reprod Immunol. 2011 Dec;92(1-2):97-102.

395 Wang XQ, Zhou WJ, Hou XX, Fu Q, Li DJ. Trophoblast-

396 derived CXCL16 induces M2 macrophage polarization that in turn inactivates NK cells at the 397 maternal-fetal interface. Cell Mol Immunol. 2018 Dec;15(12):1038-1046

399 Xu Y, Romero R, Miller D, Kadam L, Mial TN, Plazyo O, Garcia-Flores V, Hassan SS, Xu Z, 400 Tarca AL, Drewlo S, Gomez-Lopez N. An M1-

401 like Macrophage Polarization in Decidual Tissue during Spontaneous Preterm Labor That Is 402 Attenuated by Rosiglitazone Treatment. J Immunol. 2016 Mar 15;196(6):2476-2491.

404 Zeldovich VB, Bakardjiev AI. Host defense and tolerance: unique challenges in the placenta.

405 PLoS Pathog. 2012;8(8):e1002804

406

407 Zhang YH, He M, Wang Y, Liao AH. Modulators of

408 the Balance between M1 and M2 Macrophages during Pregnancy. Front Immunol. 2017 Feb

$4099 ; 8: 120$ 
Figure Legends:

411 Fig. 1. Perforin-2 limits L. monocytogenes infection of the placenta and fetus. Pregnant

412 Perforin-2 (P2) +/+ and -/- mice were infected intravenously on GD 12.5 with $2.5 \times 10^{5}$ CFU of

413 L. monocytogenes for 44 hours. Bacterial loads were then determined in each individual liver,

414 placenta, and fetus by CFU assay. Shown are the compiled results of two separate infection

415 experiments using a total of 3 pregnant mice per genotype harboring a total of either 22 FPUs (P2

$416+/+)$ or 17 FPUs (P2 -/-). Mann-Whitney U test used to calculate p-values.

418 Fig. 2: Both maternal- and fetal-encoded Perforin-2 contribute to protection of the placenta

419 and fetus. A heterogenic mating strategy was used to generate pregnant mice that possessed

420 fetuses that were either Perforin-2 (P2) +/- or -/-. Pregnant mice (GD 12.5) were infected

421 intravenously with $2.5 \times 10^{5}$ CFU L. monocytogenes for 44 hrs. (A) A representative cross is

422 shown between a $P 2$-/- female and a $P 2+/-$ male. Following infection, FPUs $(\mathrm{N}=10)$ were

423 dissected, bacterial loads determined in each individual placenta and fetus by CFU assay, and

424 individual fetuses genotyped. In the example shown, fetuses \#1-4 are $P 2+/-$ and \#5-10 are $P 2$-/-

425 . Asterisk denotes CFU values below detection limit. (B) Compiled analysis of crosses between

426 P2 -/- females, $\mathrm{N}=5$ and $P 2+/-$ males in which FPUs lack maternal-derived P2, designated as

427 M(-/-). Placentas associated with $P 2$ +/- fetuses (e.g., fetuses 1-4 in (A)) possess trophoblasts

$428 \mathrm{~T}(+/-)$ with fetal-derived P2. Placentas associated with $P 2$-/- fetuses (e.g., fetuses 5-10 in (A))

429 possess trophoblasts T(-/-) that lack fetal-derived P2. (C) Similar compiled analysis of crosses

430 between $P 2$ +/- females, N=5 and P2 -/- males in which FPUs possess maternal-derived P2,

431 designated as $M(+/-)$. Placentas associated with $P 2+/-$ fetuses possess trophoblasts, designated

432 as $\mathrm{T}(+/-)$, with fetal-derived P2. Placentas associated with $\mathrm{P} 2$-/- fetuses possess trophoblasts, 
433 designated as T(-/-), that lack fetal-derived P2. Compiled data drawn from three independent

434 infections performed on separate days. Mann-Whitney U test was used to calculate p-values. (NS

$435=$ not significant)

437 Fig. 3: Composition of immune cells in liver and decidua of uninfected and infected GD12.5

438 pregnant mice. Pregnant mice (GD 12.5) were either uninfected or intravenously infected with 1

$439 \times 10^{6}$ CFU of L. monocytogenes for $44 \mathrm{~h}$. Single-cell preparations of the indicated tissue were 440 analyzed by flow cytometry for expression of immune- and myeloid-specific markers. (A) The

441 percentage of cells staining positive for the immune-specific cell surface marker CD45 per $10^{6}$

442 isolated total live liver cells shown from an uninfected and infected mouse. The left panel shows

443 the staining profile of CD45-staining liver cells from an individual uninfected dam (top) and an

444 individual infected dam (bottom). The right panel shows the expression levels of myeloid-

445 specific markers CD11b and F4/80 of the CD45 ${ }^{+}$cells. (B) For the same mice, the percentage of

446 cells staining positive for the immune-specific cell surface marker CD45 per $10^{6}$ isolated total

447 live decidual cells are shown. The left panel shows the staining profile of CD45-staining

448 decidual cells from an individual uninfected dam (top) and an individual infected dam (bottom)

449 and the right panel shows the expression levels of myeloid-specific markers CD11b and F4/80 of 450 the $\mathrm{CD}_{4} 5^{+}$cells. Shown is a representative mouse from 3 mice per group.

452 Fig. 4: Perforin-2 expression is induced in decidual and splenic immune cells following

453 infection Pregnant BALB/c mice (GD12.5) were infected intravenously with 5 x $10^{5}-1 \times 10^{6}$ CFU 454 of L. monocytogenes for 44 hrs and decidual and splenic cells were analyzed for P2 mRNA levels. 
455 (A) Representative contour plots of background (FMO), uninfected, and infected cells gated on 456 live, $\mathrm{CD}^{+} 5^{+}$cells expressing AF-647 (P2). Histogram overlay of uninfected (red) and infected

457 (blue) $\mathrm{CD}_{4} 5^{+}$decidual cells showing Perforin-2 (P2) mRNA levels. (B) Compiled analysis of P2 458 percentage and mean fluorescence intensity (MFI) of $\mathrm{P} 2$ expression on $\mathrm{CD}_{4} 5^{+}$cells in decidua and 459 spleen. Compiled data drawn from two independent infections performed on separate days. 460 Student's T-test was used to calculate p-values.

461

Fig. 5: Specific Perforin-2 induction in decidual and splenic macrophages of BALB/c and

464 were infected intravenously with $5 \times 10^{5}-1 \times 10^{6}$ CFU of L. monocytogenes for 44 hrs and decidual and splenic macrophages were analyzed for Perforin-2 (P2) mRNA levels. (A,D) Representative contour plots of background (FMO), uninfected, and infected cells gated on live macrophages expressing AF-647 (P2). Histogram overlay of uninfected (red) and infected (blue) CD45 decidual macrophages showing P2 mRNA levels. Compiled analysis of $P 2$ percentage and mean

471 test used to calculate p-values.

473 Fig. 6: Divergent Perforin-2 expression in decidual and splenic macrophages following lower-

474 dosed infections. Pregnant BALB/c (A-C) or C57BL/6 (D,E) mice (GD 12.5) were infected

475 intravenously with $2.5 \times 10^{5}$ CFU of L. monocytogenes for 44 hrs and single-cell preparations of 476 decidual and splenic macrophages were analyzed for Perforin-2 (P2) mRNA levels and shown as 477 described in Fig. 5. Student's T-test used to calculate significance. (NS = not significant) 
479 Fig. 7: Decidual macrophages polarize to a M1 phenotype following high-dose infection.

480 Pregnant mice (GD 12.5) were either uninfected or intravenously infected with 1 x $10^{6}$ CFU

$481 \quad\left(\mathrm{PD}_{100}\right)$ of L. monocytogenes for $44 \mathrm{~h}$. Single-cell preparations of deciduas were analyzed by flow

482 cytometry for expression of M1 and M2 macrophage-specific markers. (A) Gating strategy used

483 to define macrophages (single, live, $\mathrm{CD}^{+} 5^{+}, \mathrm{CD} 11 b^{+}, \mathrm{F} 4 / 80^{+}, \mathrm{Ly}^{-}, \mathrm{Ly}^{-} \mathrm{G}^{-}$). Gating strategy and

484 proportions of $\mathrm{M} 2\left(\mathrm{CD}^{206}{ }^{+}, \mathrm{MHCII}^{\mathrm{lo}}\right)$ and $\mathrm{M} 1$ (CD206-, MHCII $\left.{ }^{\mathrm{hi}}\right)$ macrophages in a

485 representative uninfected (B) and $\mathrm{PD}_{100}$ infected (C) dam (GD 12.5). (D) Compiled analysis of

486 M1 and M2 decidual macrophages by percentage in uninfected and PD 100 infected dams. Results

487 based on 4 mice analyzed on several different days. Student's T-test was used to calculate p-values.

Fig. 8: Perforin-2 is expressed preferentially in decidual M1 macrophages. Decidual cells

490 were isolated from uninfected pregnant Perforin-2 (P2) +/+ BALB/c mice (GD 12.5) and M1

491 and M2 macrophages were analyzed for P2 mRNA. (A) Representative contour plots are shown

492 of background (FMO) and uninfected M1 (top) and M2 (bottom) macrophages expressing AF-

493647 (P2). Histogram overlay plots of individual dams showing P2 mRNA levels in M1 (clear)

494 and M2 (grey filled) decidual macrophages. (B) Compiled analysis of P2 mRNA levels in M1

495 and M2 macrophages by percentage and mean fluorescence intensity (MFI) in decidua. Results

496 based on 5 uninfected dams. Student’s T-test used to calculate p-values. 
Fig. 1

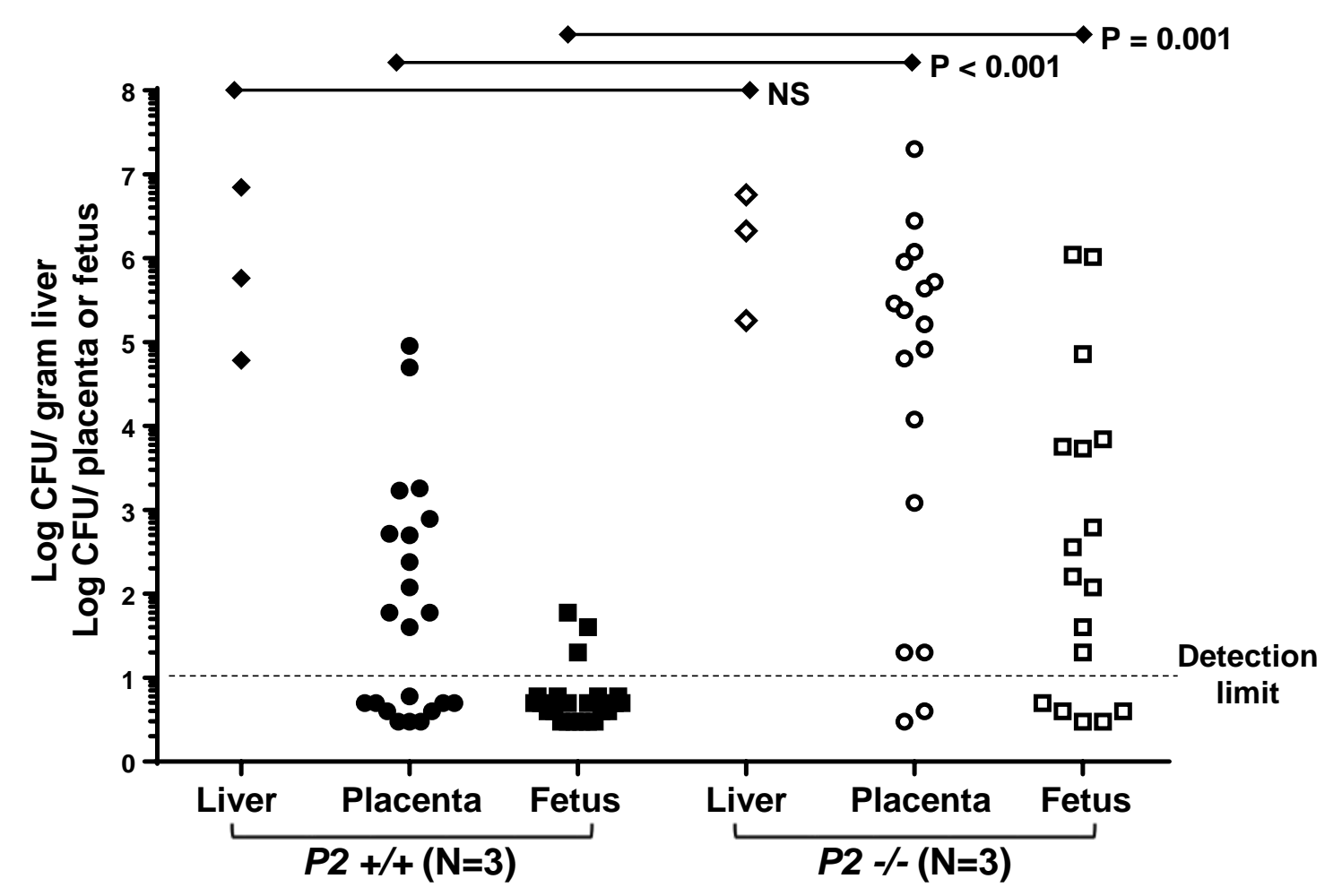


Fig. 2

A. $\quad P 2-I-($ Dam) $\times$ P2 +I- (Sire)

B. $\quad P 2-I-(\mathrm{Dam}) \times P 2+I-$ (Sire)

C. $\quad P 2+I-($ Dam) $\times P 2-I-$ (Sire)
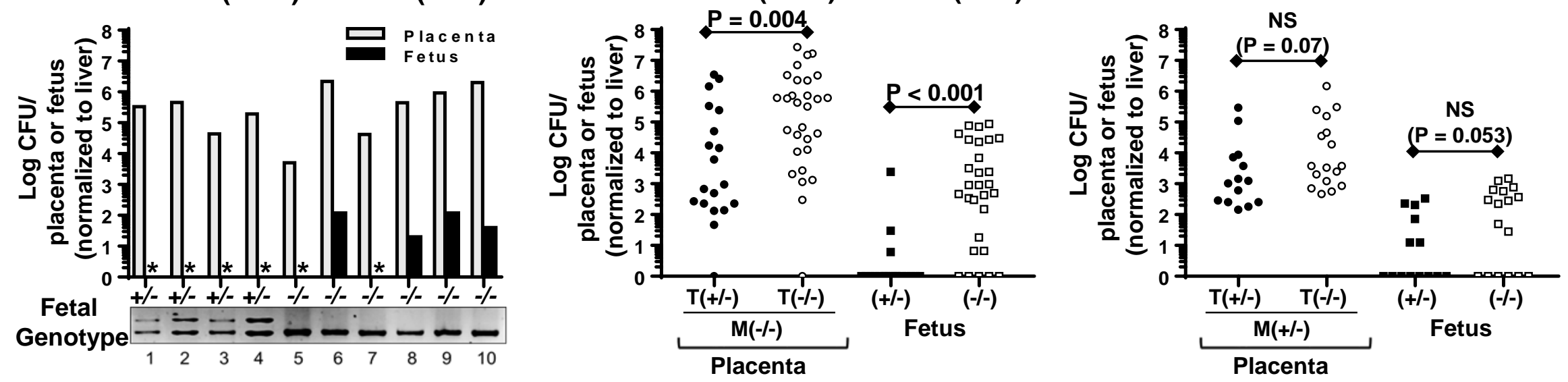
Fig. 3
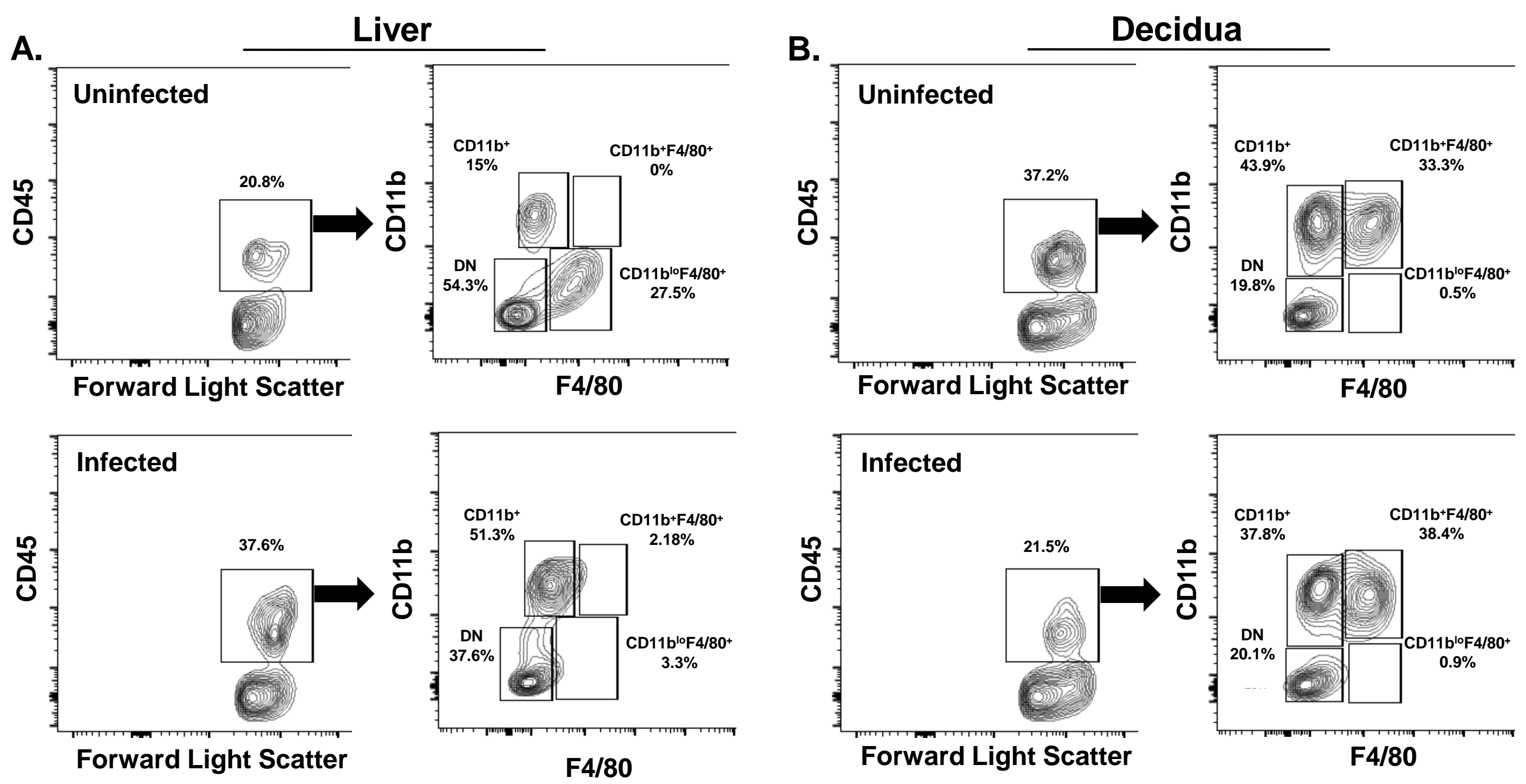
Fig. 4

A.

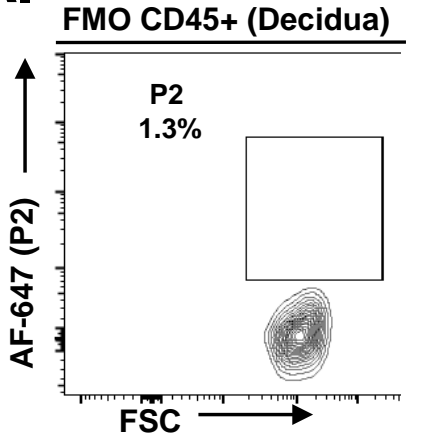

Infected CD45+ (Decidua)

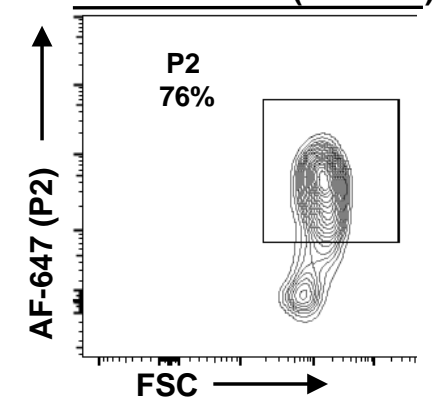

B.

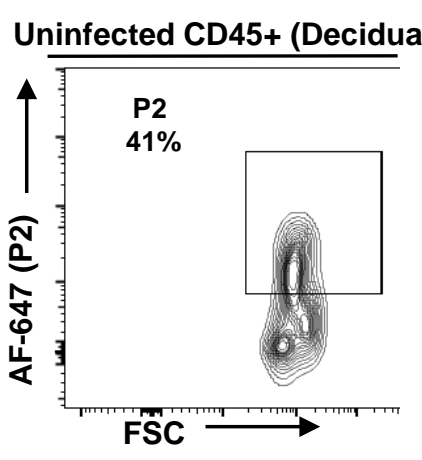

Uninfected vs Infected

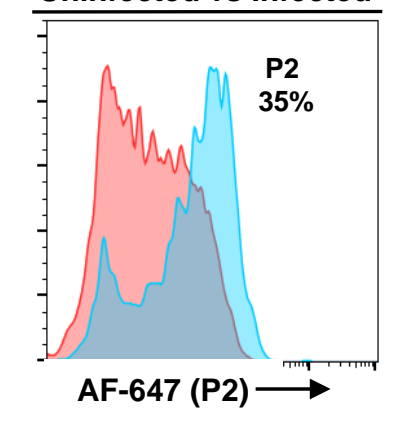

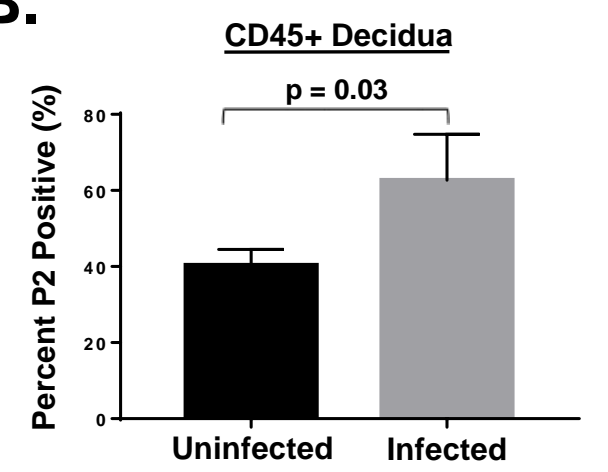

$(\mathrm{N}=3)$

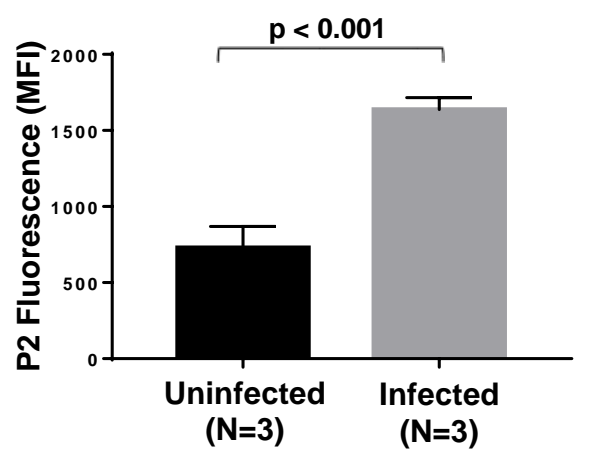

C.
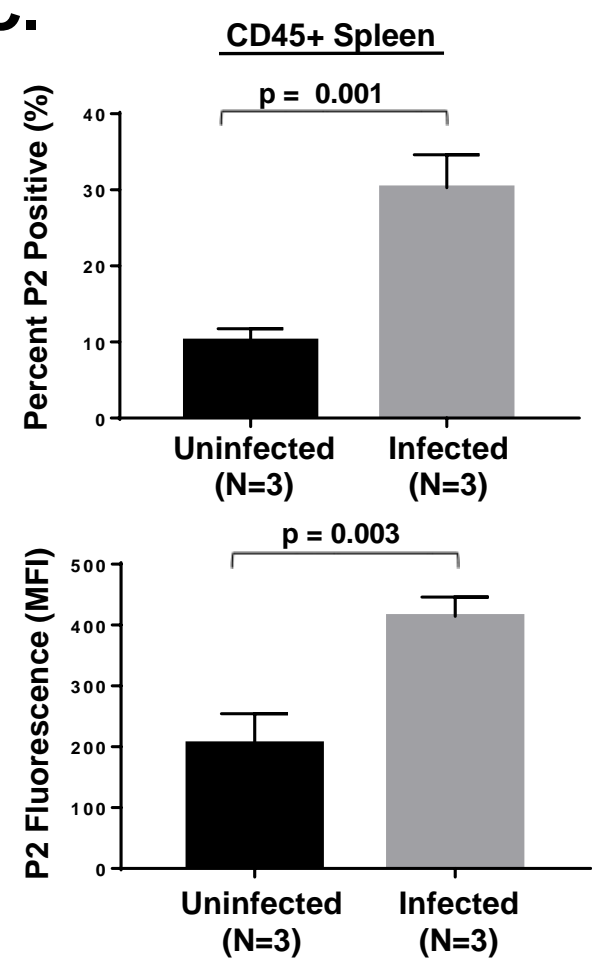
Fig. $5 A, B, C$
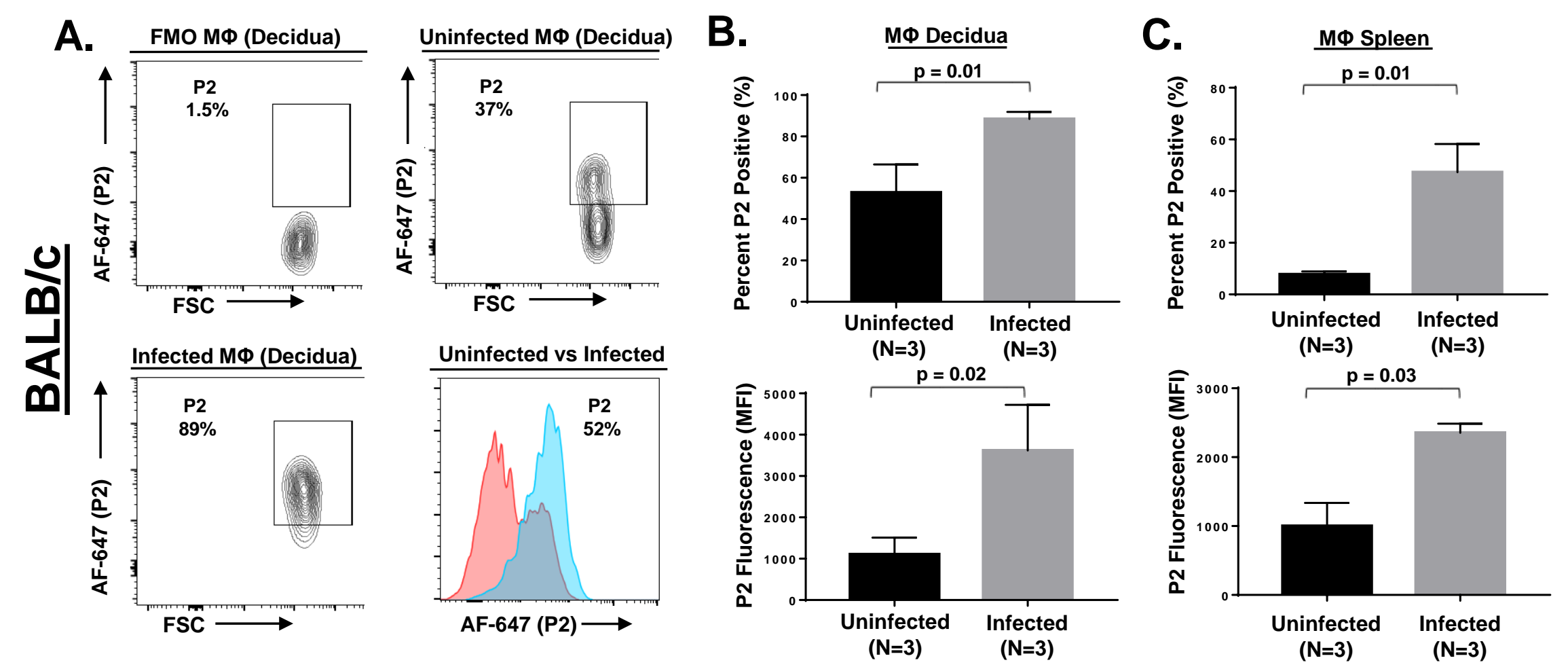
Fig. 5D,E,F
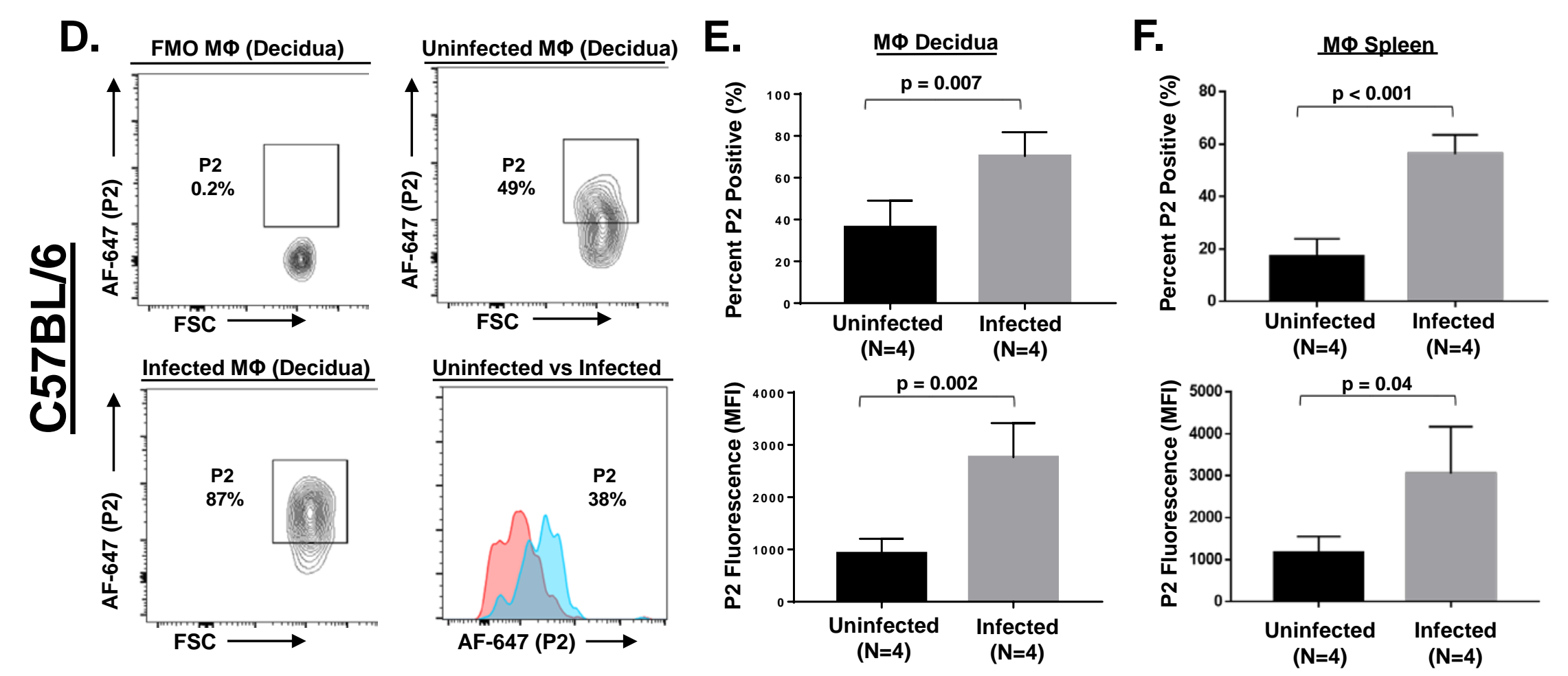
Fig. 6

A.

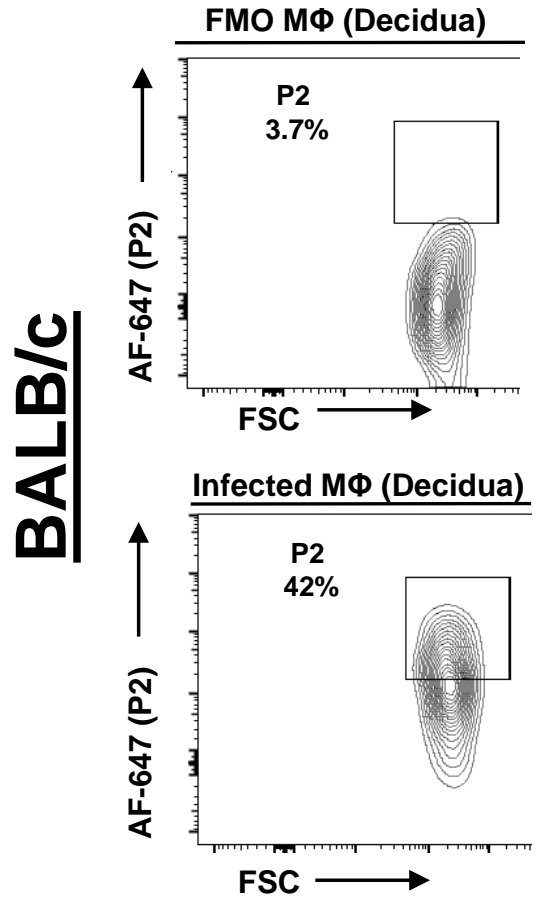

B.

Uninfected MФ (Decidua)

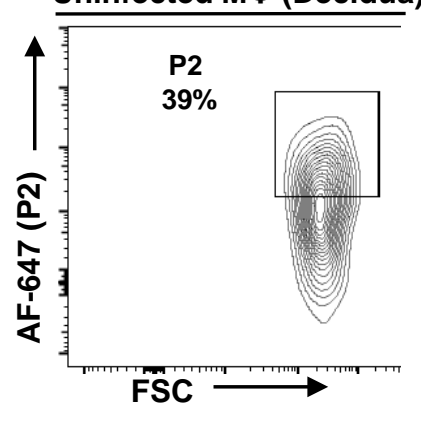

Uninfected vs Infected

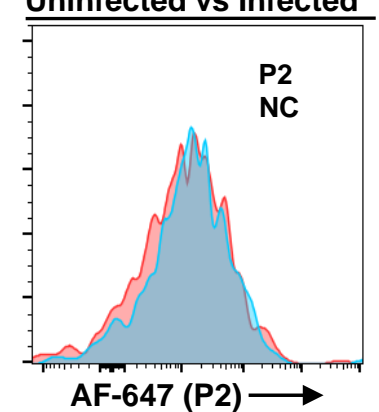

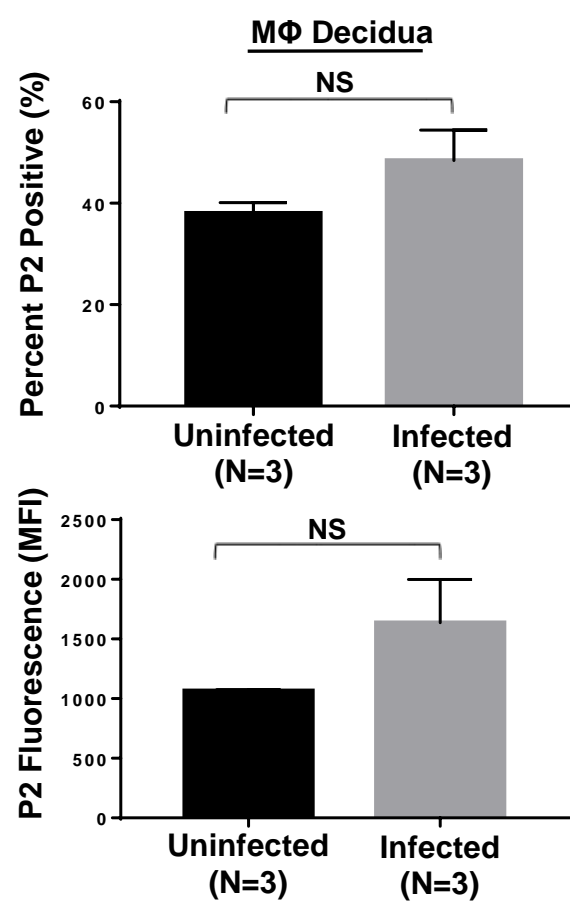

D.

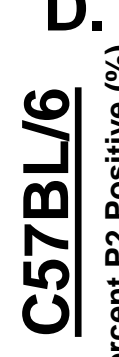

MФ Decidua NS

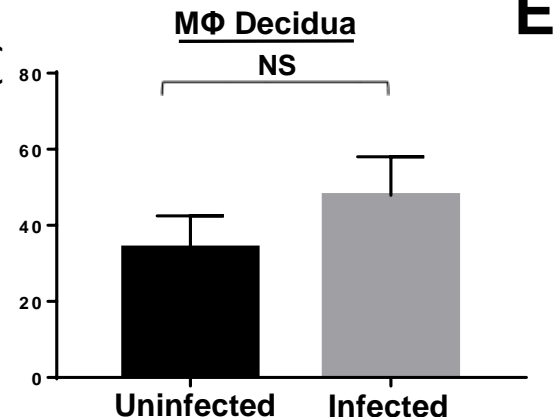

Uninfected Infected
C.

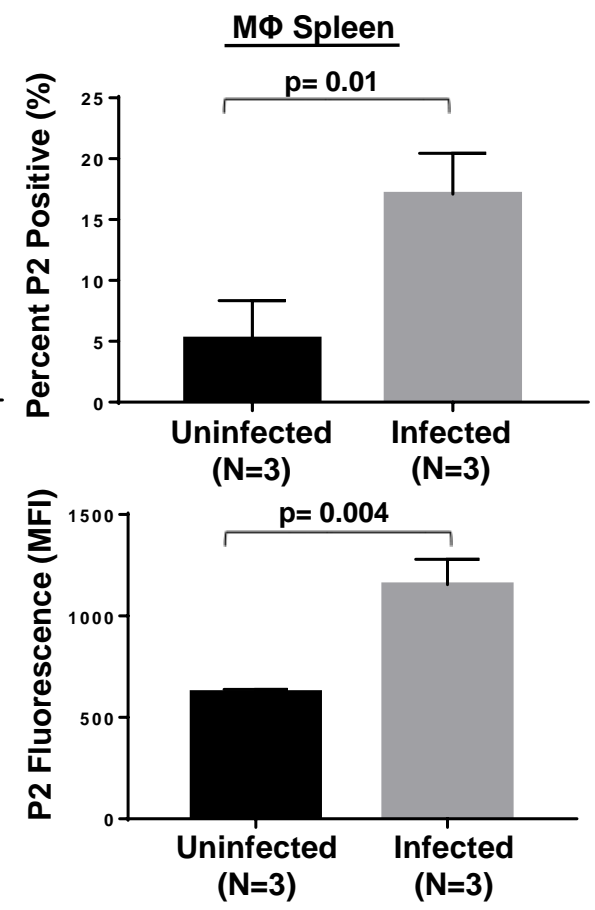

E.

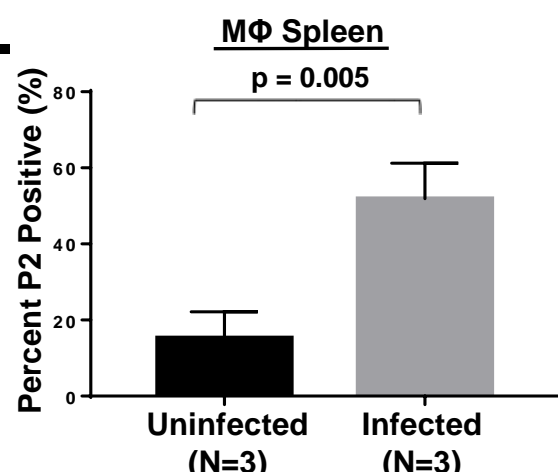


Fig. 7 A.

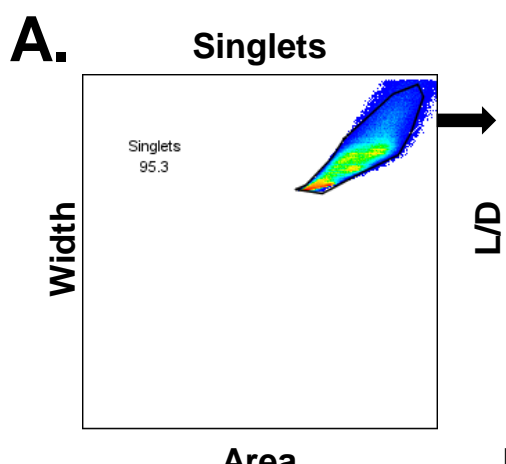

Area
Live

Forward Light Scatter

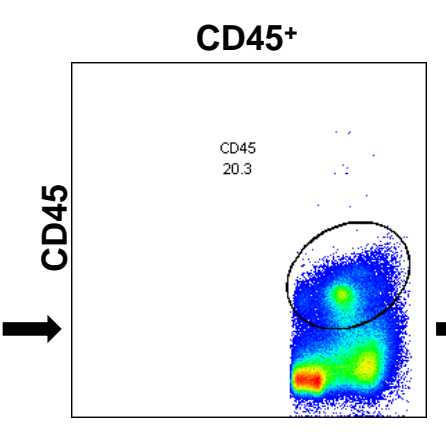

Forward Light Scatter

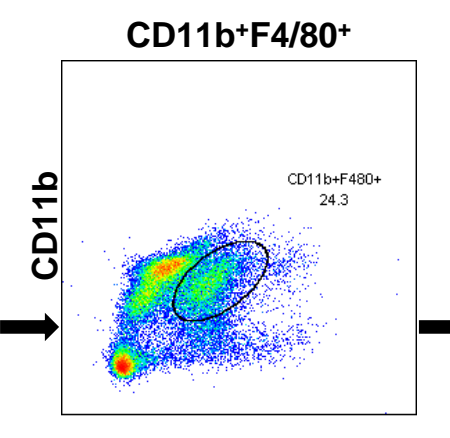

$F 4 / 80$
Ly6C-Ly6G

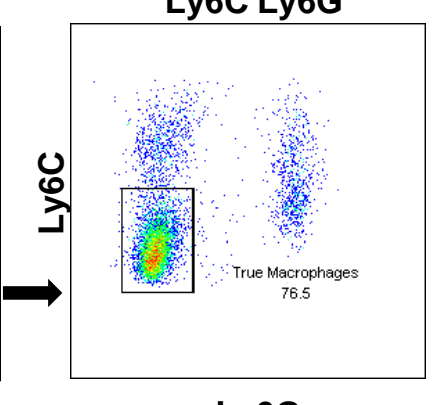

Ly6G
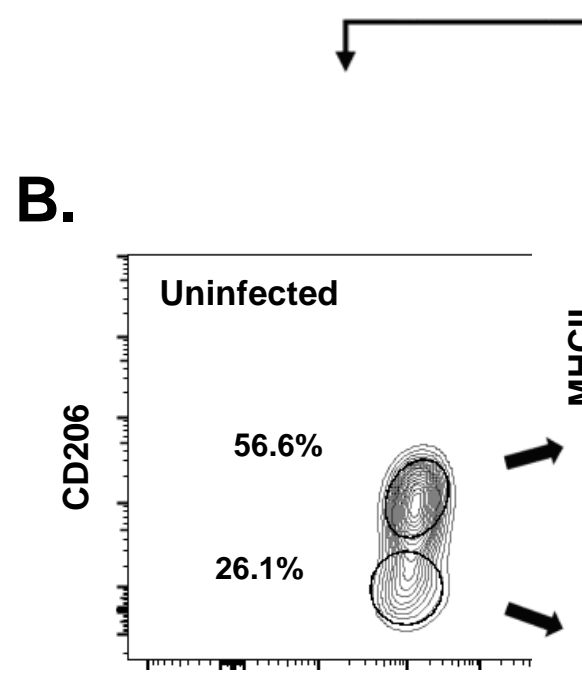

$206^{+} \mathrm{MHClI}^{\mathrm{lo}}(\mathrm{M} 2)$ Forward Light Scatter

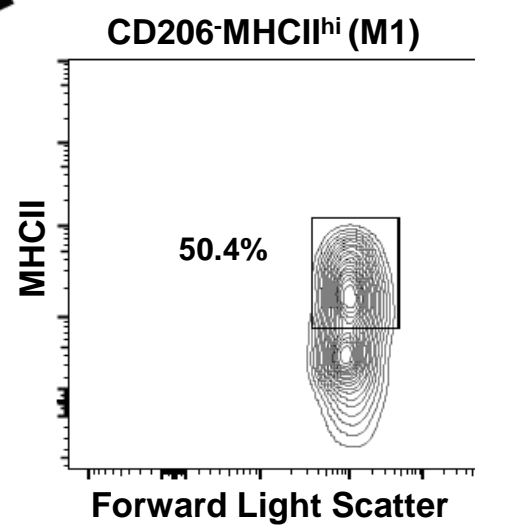

C.
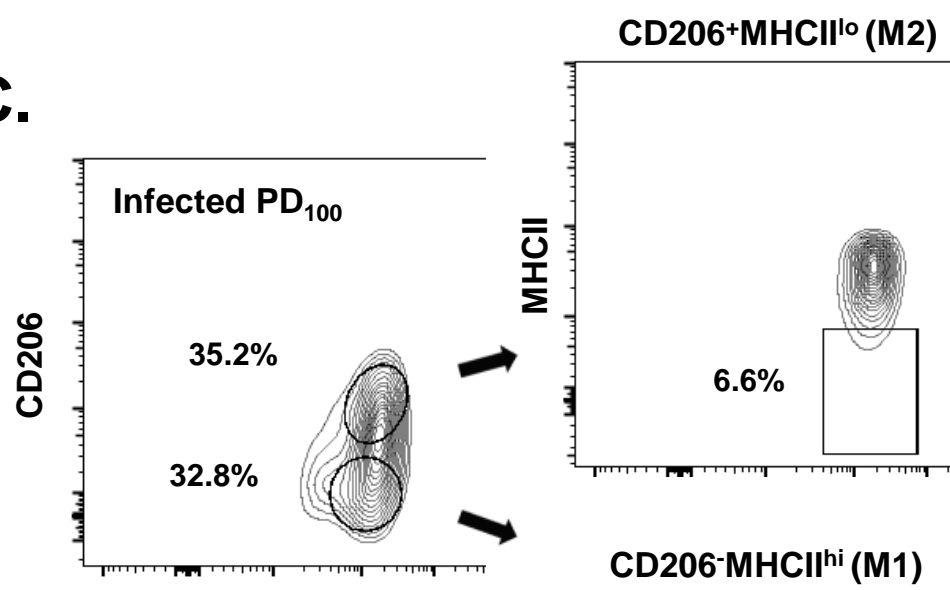

Forward Light Scatter

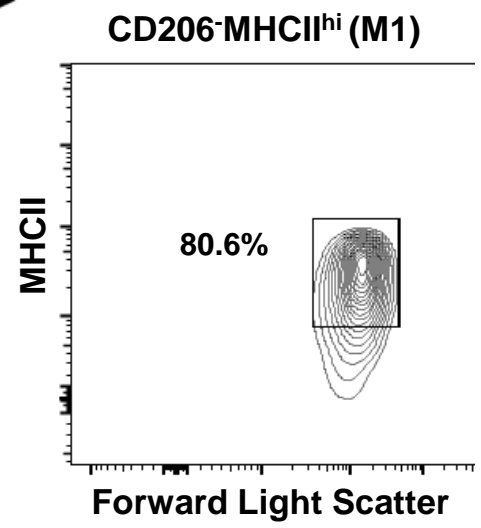

D.

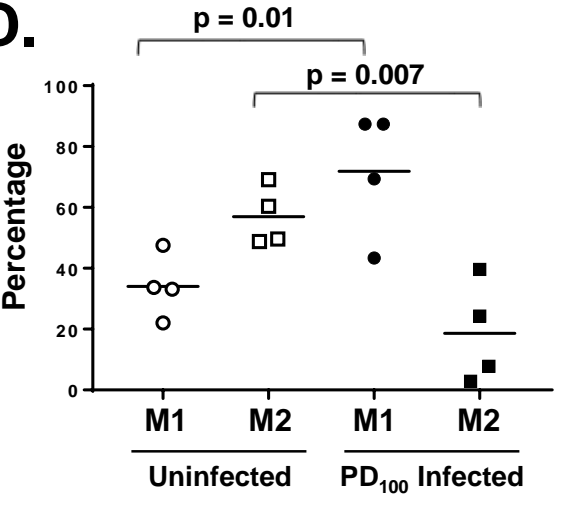


Fig. 8

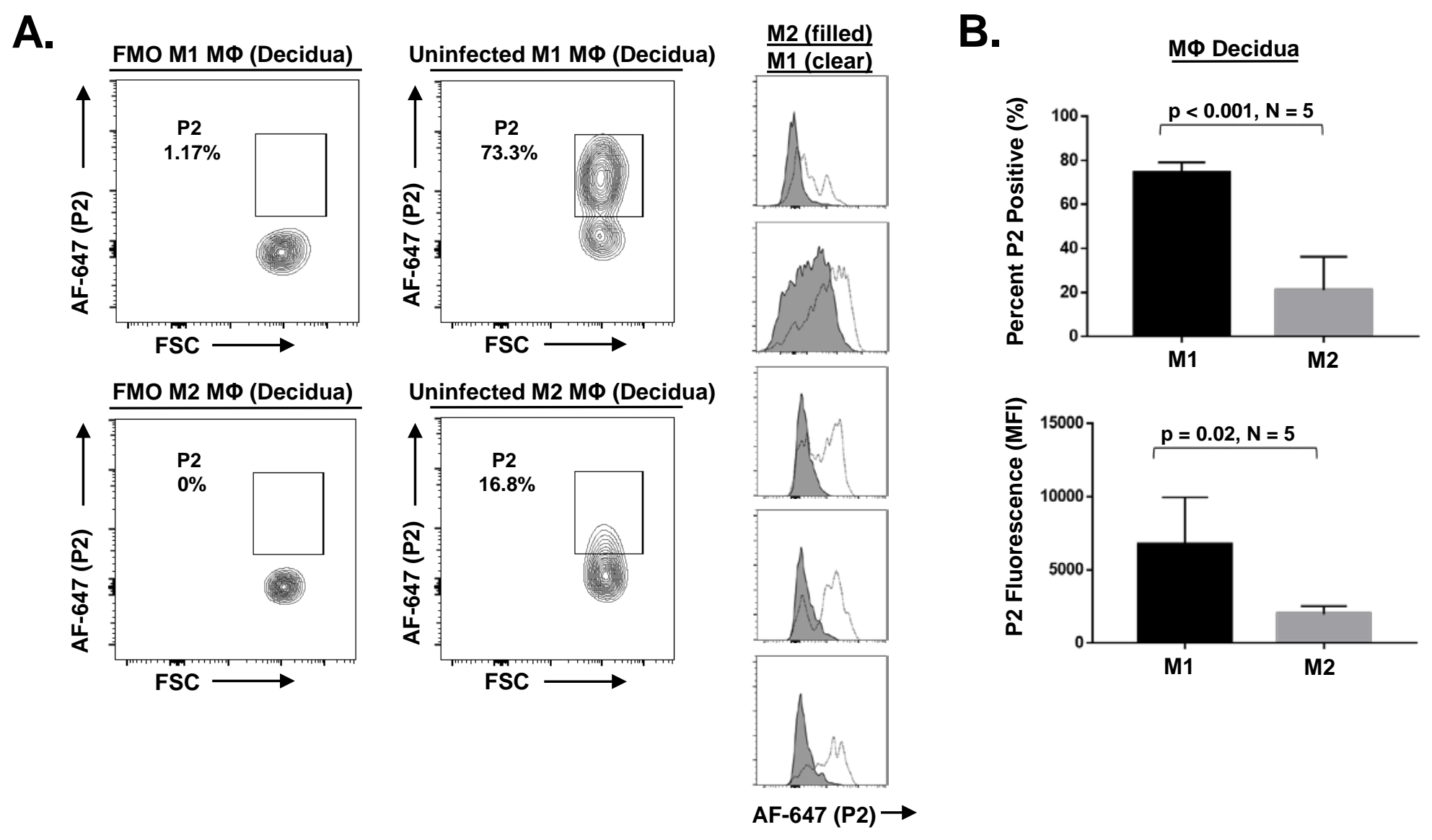

